\title{
The Physical Structure of NGC 2440
}

\section{Cuesta ${ }^{1}$ and J. P. Phillips ${ }^{2}$}

${ }^{1}$ Instituto de Astrofísica de Canarias, La Laguna;

${ }^{2}$ Instituto Nacional de Astrofísica, Óptica y Electrónica, Mexico

We have acquired monochromatic imaging of the bipolar outflow source NGC 2440 in 12 ionic transitions using the $1.5 \mathrm{~m}$ Telescope at the Observatorio de San Pedro Mártir, Ensenada, Baja California, Mexico. As a result, we have been able to undertake detailed analysis of the shell ionisation structure. Then, we found that excitation is high close the center, extending towards the exterior at located radial positions. Edges and ansae are zones of low excitation indicating probably shock regions, in particular the outer shell.

Extinction appears extremely patchy, with higher values located towards the south of the nucleus implying variations of $\sim 1 \mathrm{mag}$. On the other hand, electron density declines strongly away from the nucleus, and also shows evidence for rapid variability and irregular structure. It is particularly interesting the evidence for low density values associated to the ansae and, in general, to the low excitation regions. Finally, electron temperatures present slightly variations in structure with typical values of $1.510^{4} \mathrm{~K}$.

Evidence is presented for at least three phases of collimated mass-loss characterised by differing ejection axes. Each separate bipolar flow appears also to have been associated with symmetrically disposed low excitation ansae, of which five are present. As a result, it is proposed that we may be witnessing a re-orientation of the collimation zone, deriving from precession in a central disk.

\section{REFERENCES}

Baessgen, M., Diesch, C., and Grewing, M., 1995, Astron. Astrophys., 297, 828.

Condal, A. R., 1982, Astron. Astrophys., 112, 124.

Louise, R., and Pascoli, G., 1985, Astron. Astrophys., 150, 285. 